\title{
The genetics of inherited retinal disorders in dogs: implications for diagnosis and management
}

This article was published in the following Dove Press journal:

Veterinary Medicine: Research and Reports

15 March 2016

Number of times this article has been viewed

\section{Anna Palanova \\ Department of Tumor Biology, Institute of Animal Physiology and Genetics, v. v. i., Academy of Sciences of the Czech Republic, Libechov, Czech Republic}

Abstract: Dogs are affected by many hereditary diseases just as humans are. One group of these diseases comprises of retinal disorders, which are a growing problem in canine breeding. These disorders are heterogeneous, with diverse causative mutations and modes of inheritance. Some affect only one breed, while others may affect many breeds; some breeds are affected by only one disease, while others can be affected by two or more. Dog breeders should take into account the presence of any deleterious alleles when choosing parents for the next generation.

Keywords: hereditary, retinal, disease, dog

\section{Introduction}

As of August 2015, a total of 653 disorders/traits were found in dogs, of which 263 were Mendelian traits, 193 Mendelian traits with a known key mutation, and 357 potentially usable models for human disease. Of these, 39 diseases affect the retina; some have a known genetic basis, while others are not yet known. ${ }^{1}$

\section{Canine Leber Congenital Amaurosis}

Canine Leber congenital amaurosis (CLCA; formerly known as congenital stationary night blindness or CSNB) is one of the retinal diseases that recorded the largest shift. From finding the causative mutation through the introduction of genetic testing to gene therapy, very encouraging results were noted.

CLCA is a congenital disease (night blindness) with various degrees of visual impairment under photopic illumination (vision of affected dogs ranges from normal day vision to profound day blindness). ${ }^{2}$ The only breed affected by this disease is the Briard. Signs of disease occur early in the life of the affected $\operatorname{dog}\left(\sim 5-6\right.$ weeks). ${ }^{3}$ The causative mutation - deletion of four bases (AAGA) - of this disease was found in the RPE65 (retinal pigment epithelium-specific $65 \mathrm{kDa}$ protein) gene. ${ }^{4}$ The result of the deletion is a frame shift and this leads to a premature stop codon. ${ }^{5}$ Some experimental replacement therapies to restore retinal function have been conducted with very encouraging results. Subretinal injections of recombinant adeno-associated virus (rAAV) were performed in affected dogs and improvements in visual behavior and electroretinographic (ERG) responses were observed. Visual improvement was long term; some improvement was found in younger and older dogs ( $\sim 30$ months of age). ${ }^{6-9}$ In another study, a dog treated at the age of 30 months did not recover vision or retinal function. ${ }^{10}$ This suggests that there might be a preferred age for successful treatment with gene replacement therapy. ${ }^{10}$ The next question was whether visual
Department of Tumor Biology, Institute of Animal Physiology and Genetics, v. v. i.,Academy of Sciences of the Czech Republic, Rumburska 89, 277 21, Libechov, Czech Republic

$\mathrm{Tel}+420315639588$

Fax +420315639510

Email hrdlicova@iapg.cas.cz
Veterinary Medicine: Research and Reports 20|6:7 4I-5I

(c) (1) (8) ( 2016 Palanova. This work is published and licensed by Dove Medical Press Limited. The full terms of this license are avaiable at https://wwr.dovepress.com/terms.php

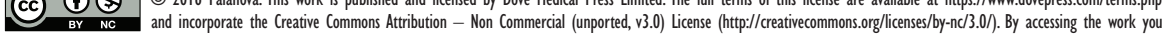
hereby accept the Terms. Non-commercial uses of the work are permitted without any further permission from Dove Medical Press Limited, provided the work is properly attributed. For peminsion for commercial use of this work, please see paragraphs 4.2 and 5 of our Terms (https://www.dovepress.com/terms.php).
Dovepress

http://dx.doi.org/10.2147/VMRR.\$63537

\section{4}


pathways leading from the defective retina to the visual cortex are intact. Therefore, RPE65-mutant dogs were studied using functional magnetic resonance imaging, and it was found that these dogs have markedly diminished retinal and subcortical responses to light. ${ }^{11}$ After treatment with rAAV, there was rapid and long-term restoration of cortical response. Human patients with RPE65 mutation (RPE65-LCA) have a preserved visual pathway and detectable cortical activation. Results from animal studies support the potential for human gene therapy to restore vision in congenitally blind patients with genetic retinal disease. ${ }^{11}$

\section{Central stationary night blindness}

True naturally occuring autosomal recessive canine central stationary night blindness (CSNB) was identified in Beagle dogs in Japan. ${ }^{12}$ Affected dogs had normal daylight vision and normal retinas but absent night vision, and they showed no detectable rod responses. The phenotype of this canine blindness is similar to the human Schubert-Bornschein form of complete CSNB. Most known candidate CSNB genes were excluded as being causative for this new form of canine blindness. The authors found reduced expression of three genes (GNAT1, CACNA2D4, and NYX) in both carriers and affected dogs; moreover, one gene (CACNA1F) was downregulated only in the affected dogs. Ongoing studies aim to clarify the exact mechanism of night blindness, identify the causative gene, and understand the neuronal plasticity and retinal remodeling that occurs in the disease. ${ }^{12}$

\section{Progressive retinal atrophy}

Progressive retinal atrophy (PRA) is a group of canine retinal degenerations. This group is very heterogeneous because some of these diseases occur early while others occur late; some are recessive, others dominant, and others X-linked. ${ }^{13}$ But all these diseases show phenotypic similarities to retinitis pigmentosa (RP), a common cause of inherited blindness in humans. ${ }^{14}$ PRAs are rod-cone dystrophies wherein rod photoreceptors (PRs) are affected earlier than cone photoreceptors. The earliest clinical sign of the disease is night blindness. ${ }^{15}$

\section{Rod-cone dysplasia I (rcd I)}

The first retinal atrophy with mutation found was rod-cone dysplasia 1 (rcd1) in Irish Setter dogs. Rcd1 is a recessive trait in which PRs are rapidly lost. Farber et $\mathrm{al}^{16}$ found that affected dogs of this breed have elevated levels of retinal cyclic guanosine monophosphate (cGMP) resulting from deficient rod-specific cGMP phosphodiesterase (cGMP PDE) activity. Other researchers performed a study of affected and putative heterozygote Irish Setters and found that the affected $P D E \beta$ subunit mRNA contained a nonsense mutation on codon 807 ( $G$ to A transition converting TGG to TAG), which led to a premature stop codon. Polymerase chain reaction (PCR) studies with genomic DNA confirmed that normal, heterozygous, and affected dogs carry the expected alleles. ${ }^{17}$ The mutation was confirmed in Setters in the UK, and a fast PCR-based diagnostic test was developed. ${ }^{18}$ Finding treatment that would slow progression of the disease is a major goal of research on hereditary blindness. The calcium channel blocker D-cis-diltiazem, which in naturally occurring PDE6B murine model of RP alters the abnormal cellular mechanisms resulting from a mutant gene causing retinal degeneration, failed to repair PRs in affected Irish Setters. ${ }^{19}$

\section{Generalized PRA in Sloughi dogs}

Generalized PRA (gPRA or rcd1a) in Sloughis has an early onset similar to that in Irish Setters and is inherited in an autosomal recessive manner. As in the Irish Setters, in Sloughis too, the causative mutation for hereditary blindness was found in the $P D E 6 B$ gene - an eight base pair (bp) insertion (TGAAGTCC) after codon 816, which causes a premature stop codon and truncates the PDE6B protein by 40 residues. $^{20}$

\section{Rod-cone dysplasia type 2}

Rod-cone dysplasia type $2(\mathrm{rcd} 2)$ is an early-onset autosomal recessive form of PRA and is phenotypically similar to LCA, an early-onset form of RP. This disease segregates naturally in the Collie breed of dogs. Kukekova et $\mathrm{al}^{15}$ mapped $\mathrm{rcd} 2$ to CFA7, an orthologue of human 1q32. Canine sequences corresponded to those of human $C R B 1$ gene; a polymorphic microsatellite was identified in intron 5 of $C R B 1$ and was used as a marker to test for cosegregation between $C R B 1$ and rcd2, but based on linkage and RH (radiation hybrid mapping) data, this gene was excluded as a candidate for $\operatorname{rcd} 2 .^{15}$ The canine gene (named RD3) homologous to C1orf36 was identified, and a 22 bp insertion that causes a frame shift and continues the open reading frame beyond the normal stop codon was found. ${ }^{21}$

\section{PRA in the Cardigan Welsh Corgi $(\operatorname{rcd} 3)$}

Rcd3 in the Cardigan Welsh Corgi is inherited in an autosomal recessive manner and has early onset leading to blindness in young adult dogs similar to that from red1. After the earlier-mentioned causative transition in the $P D E 6 B$ gene, the causative mutation (second in the dog) - the deletion 
of $1 \mathrm{bp}$ in codon 616 of PDE6A - was found in a Cardigan Welsh Corgi. ${ }^{22}$ The test for screening of the mentioned polymorphism was developed - the single-strand conformation polymorphism (SSCP) analysis. ${ }^{22}$ An allele-specific PCR-based test for rapid and inexpensive detection of the mutation was developed. ${ }^{23}$ The mutation in the PDE6A gene was excluded as causative for the gPRA in the following breeds: Chesapeake Bay Retriever, Entlebucher Sennenhund, Labrador Retriever, Tibetan Mastiff, Long- (LHD) and Wirehaired Dachshund (WHD), Tibetan Terrier, Miniature Poodle, Australian Cattle Dog, Cocker Spaniel, Saarloos/Wolfshund, Sloughi, ${ }^{24}$ and Collie. ${ }^{25}$ To avoid potential mistakes in genetic testing, in Welsh Corgis, the simple mismatch-PCR-restriction enzyme digestion test has been developed. The presence of digestion sites in the PCR product in both normal and mutant alleles provided a positive control for the activity of the restriction enzyme (Hinfl). ${ }^{26}$

\section{PRA in Gordon and Irish Setter breeds - rod-cone degeneration 4 (rcd4)}

Late-onset PRA or rod-cone degeneration 4 (rcd4) was found in two Setter breeds. A frame-shift mutation was identified in the novel PRA locus, C2orf71. Because $\sim 10 \%$ of PRA cases in the Gordon Setter breed are not caused by the mentioned mutation, it seems that at least one other mutation causes PRA in this breed. ${ }^{27}$

\section{Generalized PRA in Schapendoes dog}

In the Schapendoes dog, gPRA is characterized by initially affected rod PR vision causing night blindness, which is followed by progressive loss of cone PRs that leads to total blindness. The disease haplotype was mapped to CFA20 and, even though the causative mutation was not found, an indirect DNA test was developed for gPRA testing in Schapendoes dogs based on linkage analysis data. ${ }^{28}$ The causative mutation in the newly identified CCDC66 gene was found: a $1 \mathrm{bp}$ insertion leads to a premature stop codon. ${ }^{29}$ The Schapendoes $\operatorname{dogs}^{29}$ and true null mouse provide valuable animal models to explore the precise role of the mentioned protein in retinal degeneration and the future development of a treatment for hereditary blindness. ${ }^{30}$

\section{PR dysplasia}

In Miniature Schnauzers, the form of PRA known as PR dysplasia (pd) is an early-onset retinal disease that affects both rod and cone PRs. It is transmitted in an autosomal recessive manner. ${ }^{31}$ In pd-affected dogs, a missense mutation was found (codon 82, CGA to GGA) in the phosducin (PDC) gene. However, some pd-affected dogs were heterozygotes or homozygotes of the wild-type allele, and this indicates that another mutation may be present in this breed. ${ }^{32}$

\section{Early retinal degeneration}

Early retinal degeneration (erd) was identified in Norwegian Elkhound dogs as an early-onset autosomal recessive disease corresponding to human LCA. ${ }^{33}$ In the $S T K 38 L / N D R 2$ (serine/threonine kinase 38-like protein - a protein kinase in the nuclear Dbf2-related family) gene, two mutations were found. One (a 4 bp deletion in exon 3) was, however, excluded as causative for erd. The second, the insertion of a short interspersed nuclear element (SINE) in exon 4, results in the absence of exon 4 from the mature $S T K 38 L$ mRNA, which causes dysfunction of the protein. This was the first time that the STK38L/NDR2 pathway was implicated in PR development and/or disease. This naturally occuring erd animal model will provide new insights into PR development and role of STK38L protein in other, especially neuronal, tissues. $^{34}$

\section{PRA I in Golden Retriever (PRAI)}

In the Golden Retriever breed, clinical signs of PRA were found, but one variant - progressive rod-cone degeneration (prcd) - only accounts for a small proportion of PRA cases. A novel PRA locus was identified in CFA37. In a solute carrier anion exchanger (SLC4A3) gene, a frame-shift mutation was identified. Because a large proportion of cases $(\sim 44 \%)$ remains unexplained, it seems that three or more mutations cause PRA in the Golden Retriever breed. ${ }^{35}$

\section{PRA type 3 in Tibetan Spaniel and Tibetan Terriers (PRA3)}

Most PRA cases in Tibetan Spaniels are clinically indistinguishable from other forms of PRA. The mode of inheritance seems to be autosomal recessive. The age of diagnosis is relatively late, at $\sim 5$ years of age. Insertion of a SINE was identified in FAM161A gene, a ciliary gene associated with $\mathrm{RP}$ in humans. The mutation causes exon skipping and a shift in the reading frame, which results in a premature stop codon. The insertion is not present in all affected Tibetan Spaniels and Tibetan Terriers; this suggests that PRA in these breeds is genetically heterogeneous. ${ }^{36}$

\section{PRA in Papillon and Phalène dogs}

Primary clinical signs that were found in Papillon and Phalène dogs include visual impairment in dim light. The causative mutation known to cause PRA was not found in 
the mentioned breed (or breeds in Europe). Genome-wide association and linkage studies revealed a novel PRA locus in CFA2 in which the CNGB1 (cyclic nucleotide gated channel beta 1) gene was found. In this gene, an insertion-deletion (indel) mutation ( $1 \mathrm{bp}$ deletion followed by $6 \mathrm{bp}$ insertion, causing a frame shift and hence premature stop codon) was found, and this indel was confirmed as causative for PRA in Papillon and Phalène dog breeds. ${ }^{37}$ The PRA and the causative mutation in the $C N G B 1$ gene in the Papillon breed was confirmed by a second group of authors. ${ }^{38}$

\section{PRA in Basenji}

In the Basenji breed, the adult form of PRA was observed. First, night blindness presented, which progressed to total blindness. Many affected Basenjis retain adequate daylight vision for many years, sometimes for their entire life. This phenotype is similar to pred disease, but the pred mutation was excluded as causative in the Basenji breed. In the S-antigen $(S A G)$ gene transition mutation (T-C), which changes a normal stop codon to a code for the amino acid arginine, which would result in a deduced addition of 25 amino acids, was identified. ${ }^{39}$

\section{Progressive rod-cone degeneration}

Prcd is a phenotypically degenerative disorder in which, after normal postnatal development, rods and cones degenerate both structurally and functionally; for this reason, it is classified as a late-onset disorder. ${ }^{40}$ This retinal degeneration is inherited as an autosomal recessive trait ${ }^{41}$ and was originally characterized in a Miniature Poodle. ${ }^{42}$ The pred locus was mapped to the centromeric end of canine chromosome 9 (CFA9); this region shows synteny conservation with HSA17qter - a region in which a form of human RP17 was mapped. On the basis of clinical similarities of human and canine diseases and chromosomal localization, prcd has been proposed as a locus homologue of RP17 and a suitable animal model for the mentioned disease. ${ }^{43}$ The causative mutation in the newly identified gene $P R C D$ (progressive rod-cone degeneration) was found; it is a single $\mathrm{G}$ to A transition at nucleotide 5 of the coding sequence, which causes a cysteine to tyrosine change (C2Y) at the second amino acid of the protein in the affected animals. The same mutation was found in a patient with autosomal recessive retinitis pigmentosa (arRP) from Bangladesh. The disease-causing allele was found in the following breeds or breed varieties: Australian Cattle dog, American Cocker Spaniel, American Eskimo dog, Chesapeake Bay Retriever, Chinese Crested dog, English Cocker Spaniel, Entlebucher Mountain dog, Finnish
Lapphund, Kuvasz, Lapponian Herder, Labrador Retriever, Miniature Poodle, Nova Scotia Duck Tolling Retriever, Portuguese Water dog, Swedish Lapphund, Silky Terrier, Australian Stumpy Tail Cattle dog, and Toy Poodle. ${ }^{44}$ In the Schipperke breed, a heterozygous individual was identified; this suggests that this breed may be affected with the hereditary retinal disease - prcd. ${ }^{45}$

\section{Dominant PRA}

PRA in the English Mastiff breed revealed a disease with ambiguous mode of inheritance. Examination of pedigrees showed that the majority of affected individuals had an affected parent, which indicates dominance. ${ }^{46}$ To confirm the dominant mode of inheritance, controlled outcross matings were performed. The appearance of affected offspring from the mating indicates the presence of a dominant allele. Analysis of a candidate gene, rhodopsin ( $R H O)$, identified two synonymous and one nonsynonymous substitution. The nonsynonymous substitution ( $\mathrm{C}-\mathrm{G}$ at position 11$)$, which replaces threonine with arginine (T4R), was confirmed as causal for dominant PRA in the English Mastiff. The same substitution was found in one affected Bull Mastiff, but another affected dog did not share this mutation; this suggests that in the Bull Mastiff there must be at least one other causative mutation that causes PRA. ${ }^{46}$

\section{X-linked PRA}

X-linked PRA (XLPRA) represents a spontaneous animal model of X-linked retinal degeneration in humans. It was first found in Siberian Husky dogs. ${ }^{47}$ In hemizygous males, the outer segments of rods are affected initially; then rod outer segments almost completely disappear and cone outer segment degeneration becomes apparent. Affected males begin to show clinical signs at the age of sexual maturity or young adulthood; female carriers demonstrate the rod specificity of disease. ${ }^{48}$ The disease locus was mapped to the short arm of the $\mathrm{X}$-chromosome, which is homologous to human Xp21 in which the mutation for retinitis pigmentosa 3 (RP3) was found. ${ }^{48,49}$ In canids, three microdeletions in $R P G R$ exon ORF15 (RP GTPase regulator exon open reading frame 15) were found. One of these mutations, a five-nucleotide deletion (GAGAA), was found in the Siberian Husky dog. The disease in this breed was renamed XLPRA1 to distinguish it from a second disease (XLPRA2) in mongrel dogs. The XLPRA1 mutation causes a frame shift and immediate premature stop; the same mutation was found in the Samoyed breed with a clinically similar X-linked disease. In XLPRA2, a two-nucleotide deletion (GA) was found, which results in 
a frame shift that significantly changes the deduced peptide sequence. The XLPRA2 causes a very severe phenotype. The third mutation (a three-nucleotide deletion - eliminates a glutamic acid) in red wolves does not alter the remainder of the protein and does not cause disease. ${ }^{50}$ Because genebased therapy has limitations (eg, it requires identification of the mutated gene), the use of neuroprotective agents that can rescue PRs regardless of the genetic and/or environmental causes was developed. In several rodent and large animal models, ciliary neurotrophic factor (CNTF) was successfully used for rescue of PRs. In a group of 16 affected XLPRA2 dogs, CNTF treatment was used but no significant neuroprotective effect in the XLPRA2 retina was found. On the other hand, in the $r c d l$ control group, a statistically significant rescue of PRs with CNTF was observed. This suggests that one agent cannot treat all retinal degeneration cases but can be successfully used in some of these deleterious diseases. ${ }^{51}$ Gene therapy based on AAV 2/5 vector-mediated transfer was performed, with some positive results in XLPRA1- and XLPRA2-affected dogs. The full-length human RPGRORF15 complementary DNA driven by the human G-protein-coupled receptor protein kinase 1 (hGRK1) was used. In XLPRA1, treatment was initiated before PR loss (28 weeks of age); in XLPRA2, the injections were performed at 5 weeks of age. In XLPRA1, treatment before disease onset prevented disease development; in XLPRA2, treatment arrested disease progression and the morphology of the remaining PRs was restored to normal. ${ }^{52}$

In working Border Collies in France, PRA with X-linked mode of transmission was found. The exclusion of mutations in the RPGR gene, which causes XLPRA1 and XLPRA2, suggests that this PRA in Border Collies might correspond to a new form of X-linked PRA. This new form was named XLPRA3. ${ }^{53}$

\section{Cone-rod dystrophies}

In cone-rod dystrophies, the cone system is more affected; severe loss of central vision, color vision, and photophobia are seen in affected individuals. Onset may occur in childhood or early adolescence. ${ }^{54}$

\section{Cone-rod dystrophy I (cord I or crd4)}

Cone-rod dystrophy 1 (cord1 or crd4) in Miniature Longhaired Dachshunds (MLHDs) was primarily described as rod-cone dystrophy. First ophthalmoscopically observable signs were found in affected puppies at the age of 25 weeks (bilateral patchy and mild hyperreflectivity in tapetal fundus). Over the next few months, the intensity of hyperreflectivity increased and was accompanied by attenuation of the retinal blood vessels. ${ }^{54}$ A 44 bp insertion ( A $_{29}$ GGAAGCAACAGGATG) in RP GTPase regulator-interacting protein 1 (RPGRIP1) gene was found in affected MLHDs from the research group; and 5.3\% (two individuals) of dogs from the pet population were homozygous for the mutation but without clinical signs of disease. ${ }^{55}$ To confirm or exclude the insertion as causal for cord1, dogs from a research colony expanded from the original study were used (recessive homozygotes with insertion, as well as heterozygotes) and clinical findings, ERG, objective vision testing, histology, and other tests were performed; the 44 bp insertion was consequently excluded from direct causal association with cord $1 .{ }^{56}$ Then, an additional single locus on canine chromosome 15 was found in which the majority of early-onset cases were homozygous for a $1.49 \mathrm{Mb}$ interval. It seems that homozygosity at both locuses must be present for early-onset retinal degeneration to develop. ${ }^{57}$

\section{Cone-rod dystrophy in Standard Wire- haired Dachshund}

First behavioral changes in affected puppies of Standard Wire-haired Dachshund (SWHD) breed were seen at the age of 6 weeks, but age of onset and progression of retinal degeneration showed marked diversity. Initial onset of changes was observed between 10 months and 3 years, and changes were always bilateral and symmetrical. A complete retinal atrophy was evident at the age of 5-6 years. Analysis of the ERG recordings showed severely reduced cone single-flash a- and b-wave amplitudes.$^{58}$ The mode of inheritance of cone-rod dystrophy (crd) in the SWHD is autosomal recessive. The causative mutation, deletion of $180 \mathrm{bp}$, was found in the nephronophthisis 4 (NPHP4, also known as nephroretinin) gene. The affected protein lacks the domain interacting with RPGRIP1 in retina. ${ }^{59}$

\section{Cone-rod dystrophy I and 2 (crd I and crd2)}

In American Staffordshire Terrier dogs and American Pit Bull Terrier dogs, two early-onset autosomal recessive retinal degenerations were identified. ${ }^{60}$ In both breeds, the disease affects young dogs ( $<1$ year of age). Dogs are affected with severe photopic and scotopic visual impairment, which progresses to more severe blindness in early adulthood. The disorders are similar in both breeds and so were termed crd1 and crd2, respectively. For the same reason, and because the breeds share a common ancestor and are physically similar, a cross-breeding complementation test was undertaken to 
prove that the two degenerations were nonallelic. This was confirmed, and in crd1-affected dogs, mutation in the PDE6B gene was found (three-base deletion in exon 21, which causes deletion of one amino acid - asparagine - at position 802 of the protein). In crd2-affected dogs, a 1 bp insertion in exon 10 of the IQCBI (IQ motif-containing protein B1 or nephrocystin 5-NPHP5) gene was identified, which causes a frame-shift mutation and premature stop codon. ${ }^{61}$

\section{Cone-rod dystrophy 3 (crd3) or gPRA}

In the Irish Glen of Imaal Terrier (GIT) breed of dogs, the disorder termed cone-rod dystrophy was identified. It is an adult-onset disease, with the first signs presenting in affected dogs at 3 years of age and progressing to end-stage retinal degeneration over several years. This disease is orthologous to human cone-rod dystrophy 9 (CORD9), which is caused by a mutation in the A Disintegrin and Metalloprotease domain, family member 9 (ADAM9) gene. In the same gene, in dogs, a large genomic deletion was found (it removes exons 15 and 16 from the $A D A M 9$ transcript, introduces a premature stop codon, and would remove critical domains from the encoded protein). ${ }^{62}$ The deletion was confirmed in another group of affected GIT dogs with gPRA, which indicates that crd3 and gPRA in GIT is the same disease with the same causative deletion in the $A D A M 9$ gene. ${ }^{63}$

\section{Achromatopsia}

Achromatopsia or rod monochromacy and day blindness is a rare autosomal hereditary disease that results in complete loss of cone PR function, while the rods remain intact. The disease is characterized by decreased visual acuity, photophobia, nystagmus, and complete color blindness. ${ }^{64}$ Cone degeneration (cd) in Alaskan Malamute and German Shorthaired Pointer breeds is inherited in an autosomal recessive manner and is homologous to human achromatopsia. Mutations in the canine homologue of the cyclic nucleotide-gated channel $\beta$-subunit gene ( $C N G B 3)$, responsible for human $A C H M 3$ disease phenotype, were found. In Alaskan Malamute-derived dogs, the deletion that removes all exons of the mentioned gene was found. In the German Shorthaired Pointer, a missense mutation in exon $6(\mathrm{D} 262 \mathrm{~N})$ within a conserved region of $C N G B 3$ gene was found. Affected dogs from both breeds provide a natural model for the study of disease mechanisms and development of potential therapeutic methods. ${ }^{65}$ The same deletion as in the Alaskan Malamute was found in some other breeds. Two of them were Siberian Husky and Alaskan Sled dog, which are from a subgroup of distance runners and share a common ancestor, but one other, the Miniature
Australian Shepherd, is not genetically related to the Alaskan Malamute; this suggests that other breeds may potentially carry the same cd allele and be affected by achromatopsia. ${ }^{66}$ Gene replacement therapy using rAAV serotype 5 (rAAV5) was succesfully used for restoration of cone function and associated photopic vision in both canine achromatopsia models. Results of this study hold promise for future clinical trials in human patients with $C N G B 3$ achromatopsia. ${ }^{67}$

\section{Canine multifocal retinopathy}

Best macular dystrophy (BMD) is a human autosomal dominant disease of the retina caused by mutations in the bestrophin (BEST1) gene. BMD typically presents in childhood. ${ }^{68}$ Even though dogs lack the foveomacular region affected in BMD, lesions observed in a disease termed canine multifocal retinopathy $(\mathrm{cmr})$ closely resemble the vitelliform lesions of BMD. This hereditary retinal abnormality was seen in several dog breeds. Autosomal recessive mode of inheritance was supposed after pedigree analysis and prospective matings. On the basis of similarities between BMD and cmr, the BEST1/ $V M D 2$ (canine) gene was selected for phenotype-directed candidate gene analysis. Two mutations in the coding sequence were found. The first, which causes cmrl in Great Pyrenees dogs, English Mastiff, and Bull Mastiff breeds, was a nonsense mutation in exon 2 at codon $25(\mathrm{C}-\mathrm{T})$, which replaces an arginine residue with a stop codon. The second, which causes cmr2 in the Coton de Tulear breed, was a missense mutation $(\mathrm{G}-\mathrm{A})$ in exon $5 .{ }^{69} \mathrm{In}$ dogs from the Lapponian Herder breed, a retinal disease similar to $\mathrm{cmr}$ was found, but mutations for $\mathrm{cmr} 1$ and $\mathrm{cmr} 2$ were excluded as causative for this variant of the disease. The novel form of retinopathy was named $\mathrm{cmr} 3$, and two mutations in the coding sequence of BEST1 were identified: a deletion at nucleotide position 1,388 (results in a frame shift and introduces a premature stop codon) and a substitution at nucleotide position 1,466 (leads to change in the amino acid sequence - glycine to valine, and in combination with the first-mentioned mutation, causes an additional stop codon). Mutations were found in complete linkage disequilibrium. In dogs from the Bernese Mountain dog breed, two other potentially deleterious mutations were found, but only in the heterozygous state, and no homozygous affected dog has been identified. This indicates that there may be another variant of $\mathrm{cmr}$. Causative mutation for $\mathrm{cmrl}$ was identified in the new breeds Dogue de Bordeaux and Italian Cane Corso, ${ }^{70}$ Australian Shepherd, ${ }^{71}$ and Boerboel. ${ }^{72}$ The affected dogs with cmr1 and cmr2 were used in a detailed study to characterize BEST disease in this naturally occurring large animal model. ${ }^{73}$ 


\section{Retinal and skeletal dysplasia or oculoskeletal dysplasia}

Retinal and skeletal dysplasia or oculoskeletal dysplasia (OSD) is a hereditary disease, which presents in the Labrador Retriever and Samoyed dog breeds. In this disease, normally organized retinal cells are disorganized and can form folds. In severe forms of disease, the retina can be unattached and other abnormalities can be seen in the eye (cataract). Depending on the severity of the disease, dogs may have reduced eyesight or can be totally blind. Skeletal problems include joint abnormalities, the breakage of pieces of affected bones, and stunted growth of bones in legs ${ }^{74}$ Cross-breeding of an OSD-affected Labrador Retriever to an OSD-affected Samoyed resulted in a nondwarf progeny; this established that these two disorders are nonallelic. OSD in the Labrador Retriever breed has been termed drd1 (dwarfism with retinal dysplasia type 1) and OSD in the Samoyed breed has been termed drd2 (dwarfism with retinal dysplasia type 2). In two types of collagen, causative mutations for both forms of disease were found, with drd1 cosegregating with an insertional mutation (an insertion of a guanine in exon 1) in COL9A3 gene and drd2 cosegregating with a deletion mutation (a 1,267 bp deletion in the 5 -end of the gene) in COL9A2. Both mutations are predicted to truncate the respective protein product. These naturally occurring canine models can be used to further evaluate the relationships between the COL9 alpha chains, their regulatory mechanisms, as well as future treatment possibilities. ${ }^{75}$

\section{Collie eye anomaly}

Collie eye anomaly (CEA) is a hereditary visual impairment with heterogeneous signs. Primarily, the retina is not affected, but in severe forms of the disease, retinal detachment can be present. CEA affects many dog breeds that are members of the herding group or those breeds that were used for their creation (Collie Rough and Smooth, Border Collie, Australian Shepherd, Shetland Sheepdog - Sheltie, Nova Scotia Duck Tolling Retriever, Lancashire Heeler, Longhaired Whippet, Boykin Spaniel, and the Hokkaido dog breed). Some other breeds may be affected by CEA, but the disease has not been identified in others to date. The causative mutation for CEA was identified in the nonhomologous end joining factor 1 (NHEJ1) gene as a large deletion in intron 4, which contains several highly conserved elements (reviewed by Palanova ${ }^{76}$ ).

\section{Retinal diseases with unidentified genetic basis}

The retinopathy observed in the Swedish Vallhund - based on its inheritance patterns and the progressive nature of its vision loss - appears to be a form of PRA. The phenotype of this trait is rather different from most forms of PRA (multifocal, rather than diffuse, distribution of retinal degeneration). Age of disease onset and disease progression can vary considerably. This progressive retinopathy affects both rod and cone PRs, as well as the RPE. Affected dogs do not share common alleles, and so six known canine retinal disease genes (BEST1, PDE6A, $P D E 6 B, R P E 65$, NPHP4, and CNGB3) were excluded as causative for novel PRA in Swedish Vallhunds. Because of the individual variability in disease progression, even among related dogs, it seems that genetic disease modifiers are present in this breed. ${ }^{77}$

Retinal degeneration II (central PRA or pigment epithelial dystrophy) is a disease of the PR cells and retinal blood vessels. The first sign of this disease is central vision loss. Later on in the course of the disease, cataracts may develop. ${ }^{1}$ Two genes have been associated with this disease (GNB3 [encoding guanine nucleotide binding protein or $\mathrm{G}$ protein], beta polypeptide 3, and PRPH2 [encoding peripherin 2]), but no causative mutations were found. ${ }^{78,79}$ But the majority of central PRA has been considered nutritional rather than genetic, eg, central PRA in the Walker Hounds and Beagles fed with a vitamin E-deficient diet. ${ }^{80}$

Retinal detachment was observed, eg, in the Shi-Tzu breed. Retinal and vitreous degeneration were also frequently observed in affected dogs from the mentioned breed. ${ }^{81}$

Retinal dysplasia affects many breeds, is due to the incorrect formation of the retina, and results in complete vision loss. ${ }^{1}$

Retinal dysplasia and persistent hyperplastic primary vitreous were found in Miniature Schnauzer dogs. It is a congenital hereditary trait with autosomal recessive mode of inheritance, and so far, the causative gene has not been found. ${ }^{82}$

Retinal dystrophy or choroideremia was observed in the Great Dane breed. It exhibits hereditary progressive PR degeneration. The CHM gene was screened in 22 breeds with various forms of retinal dystrophies but variants were found to be nonpathogenic. ${ }^{83}$

Retinoschisis is not simple to detect because it is often confused with retinal degeneration. The most common form is secondary retinoschisis subsequent to other disorders. ${ }^{1,84}$

Rod dysplasia is a hereditary retinal disease inherited as a recessive trait in the Norwegian Elkhound. ${ }^{1}$

Rod-cone dysplasia is supposed to be associated with GNGT2 (guanine nucleotide binding protein [G protein], gamma transducing activity polypeptide 2 ) gene, ${ }^{1}$ but altered 
localization of GNGT2 protein in affected retinas was found only in cd-affected dogs. ${ }^{85}$

\section{New findings in hereditary retinal diseases}

A number of genetic mutations for different forms of retinal degenerations were found, but key components and the molecular events that link specific mutations to PR degeneration remain poorly characterized. Multiple pathways (proapoptotic and prosurvival) are associated with PR degeneration. ${ }^{86}$ Epigenetic mechanisms (eg, microRNA [miRNA] regulation) also play an essential role in the control of the complex visual processes during eye development and disease. ${ }^{87}$ The work of Genini et $a l^{88}$ focused on expression of miRNAs in healthy and XLPRA2 (as well as rcd1, erd, and pred)-affected retinas brings forth some interesting findings. The expression of miRNAs was monitored in three phases of retinal development after birth (at 3 weeks - induction phase; 7 weeks execution phase; and 16 weeks - chronic cell death phase in affected tissue). Results demonstrated that miRNA expression differences were minimal between 3 weeks and 7 weeks, but a substantial number of altered miRNAs were identified at 16 weeks of age in XLPRA2-affected retinal tissues compared to normal healthy tissues. This suggests that miRNAs may be coeffectors or may arise in response to retinal disease progression, but they are not initiators of the PR degeneration process. Although different mutations trigger retinal diseases, the authors ${ }^{88}$ observed commonalities in the miRNA expression pattern, which appear to be associated with the kinetics of PR cell death. This suggests that the use of miRNAs as a target for future therapeutic design might be effective for treating retinal degenerative diseases regardless of the causative mutation. ${ }^{88}$

\section{Genetic testing of hereditary retinal diseases}

Many genetic tests for screening for causative mutations of hereditary retinal diseases were developed, 3,17,22,23,89 and some of them were further improved ${ }^{26,90,91}$ for faster, simple, and accurate identification of affected dogs and carriers of the mutations. For a detailed review of genetic testing of hereditary diseases of dogs, refer the work of Mellersh. ${ }^{92}$ The advantages of DNA tests are that, unlike clinical diagnostic methods, they can detect unaffected carriers and subclinical cases prior to the onset of disease. If there are other forms of retinal diseases or any other hereditary disease with unidentified mutation in the breed, currently available DNA tests are not able to exclude all inherited diseases in the breed. ${ }^{93}$

\section{Implications for diagnosis and management of hereditary retinal diseases}

Because hereditary diseases represent a growing problem in many dog breeds all over the world, it is necessary to fight them. Screening for clinical signs of retinal diseases and genetic testing of known causative mutations should be standard in breeding practice.

But not all retinal diseases and other hereditary traits have causative mutations identified. These traits should also be taken into account to assess the overall health and genetic potential of the dog. ${ }^{93}$

It is important to preserve genetic variability within and across dog breeds. One of the common problems in dog breeding is common sire phenomenon, but some breeder clubs have solved it successfully (for a detailed review focused on dog breeding problems, refer the work of Leroy ${ }^{94}$ ).

In some dog breeds, only one hereditary retinal disease presents, so it is possible to eliminate the disease allele successfully by simple elimination of all carriers of this allele (especially if the frequency of disease allele is low). In some extraordinary cases, when the affected dog (or carrier of causative mutation) is an excellent individual of the breed, breeding can be permitted; all of the progeny should be tested for the presence of the disease allele. Other breeds carry more than one hereditary retinal disease and, sometimes, other hereditary diseases with known or unknown genetic bases. This complicates the situation for breeders and breeder clubs. Generally, it is not possible to choose completely genetically healthy parents for the next generation of dogs (we are speaking of known genetically transmitted traits only, because these are what we can control). Breeders must take into account not only the genetic health of the dog, but also its good character, which is frequently neglected, and the working utilization of the dog for which the breed was created. First, the frequently occurring disease/trait should be solved, and after the reduction of incidence of this disease allele, another allele should be chosen to fight it with. The dog, man's best friend, represents not only our assistant in many facets of our lives, it is our pet, friend, and a suitable animal model of many diseases too. Dogs are dependent on our care and protection, and that is why we must take care of their health and welfare. 


\section{Disclosure}

The author reports no conflicts of interest in this work.

\section{References}

1. omia.angis.org.au [homepage on the Internet]. Sydney: OMIA - Online Mendelian Inheritance in Animals [updated September 1, 2015; cited August 25, 2015]. Available from: http://omia.angis.org.au/home. Accessed September 2, 2015.

2. Narfström K, Wrigstad A, Ekesten B, Nilsson SE. Hereditary retinal dystrophy in the Briard dog: clinical and hereditary characteristics. Prog Vet Comp Ophthalmol. 1994;4:85-92.

3. Aguirre GD, Baldwin V, Pearce-Kelling S, Narfström K, Ray K, Acland GM. Congenital stationary night blindness in the dog: common mutation in the RPE65 gene indicates founder effect. Mol Vis. 1998;4:23.

4. Gal A, Gu S, Bremser D, et al. Mutation spectrum of RPE65 in autosomal recessive childhood-onset severe retinal dystrophy. Invest Ophthalmol Vis Sci. 1998;39:S901.

5. Veske A, Nilsson SEG, Narfström K, Gal A. Retinal dystrophy of Swedish briard/briard-beagle dogs is due to a 4-bp deletion in RPE65. Genomics. 1999;57:57-61.

6. Narfström K, Katz ML, Ford M, Redmond TM, Rakoczy E, Bragadóttir R. In vivo gene therapy in young and adult RPE65-/- dogs produces long-term visual improvement. J Hered. 2003;94:31-37.

7. Ford M, Bragadóttir R, Rakoczy R, Narfström K. Gene transfer in the RPE65 null mutation dog: relationship between construct volume, visual behaviour and electroretinographic (ERG) results. Doc Ophthalmol 2003;107:79-86.

8. Narfström K, Katz ML, Bragadóttir R, et al. Functional and structural recovery of the retina after gene therapy in the RPE65 null mutation dog. Invest Ophthalmol Vis Sci. 2003;44:1663-1672.

9. Acland GM, Aguirre GD, Bennet J, et al. Long-term restoration of rod and cone vision by single dose $\mathrm{AAAV}$-mediated gene transfer to the retina in a canine model of childhood blindness. Mol Ther. 2005;12(6): 1072-1082.

10. Le Meur G, Stieger K, Smith AJ, et al. Restoration of vision in RPE65deficient Briard dogs using an AAV serotype 4 vector that specifically targets the retinal pigment epithelium. Gene Ther. 2007;14(4):292-303.

11. Aguirre GK, Komáromy AM, Cideciyan AV, et al. Canine and human visual cortex intact and responsive despite early retinal blindness from RPE65 mutation. PLoS Med. 2007;4(6):e230.

12. Kondo M, Das G, Imai R, et al. A naturally occuring canine model of autosomal recessive congenital stationary night blindness. PLoS One. 2015;10(9):e0137072.

13. Millichamp NJ. Retinal degeneration in the dog and cat. Vet Clin North Am Small Anim Pract. 1990;20:799-835.

14. Parry HB. Degenerations of the dog retina. II. Generalised progressive retinal atrophy of hereditary origin. $\mathrm{Br} J$ Ophthalmol. 1953;37:487-502.

15. Kukekova AV, Nelson J, Kuchtey RW, et al. Linkage mapping of canine rod cone dysplasia type $2(r c d 2)$ to $C F A 7$, the canine orthologue of human 1q32. Invest Ophthalmol Vis Sci. 2006;47:1210-1215.

16. Farber DB, Danciger JS, Aguirre G. The beta subunit of cyclic GMP phosphodiesterase mRNA is deficient in canine rod-cone dysplasia 1. Neuron. 1992;9(2):349-356.

17. Suber ML, Pittler SJ, Qin N, et al. Irish setter dogs affected with $\mathrm{rod} /$ cone dysplasia contain a nonsense mutation in the rod cGMP phosphodiesterase ß-subunit gene. Proc Natl Acad Sci USA. 1993;90: 3968-3972.

18. Clements PJM, Gregory CY, Peterson-Jones SM, Sargan DR, Bhattacharya SS. Confirmation of the rod cGMP phosphodiesterase $\beta$ subunit (PDEß) nonsense mutation in affected rcd-1 Irish setters in the UK and development of a diagnostic test. Curr Eye Res. 1993;12(9):861-866.

19. Pearce-Kelling SE, Aleman TS, Nickle A, et al. Calcium channel blocker D-cis-diltiazem does not slow retinal degeneration in the PDE6B mutant $r c d l$ canine model of retinitis pigmentosa. Mol Vis. 2001;7:42-47.
20. Dekomien G, Runte M, Gödde R, et al. Generalised progressive retinal atrophy of Sloughi dogs is due to an 8-bp insertion in exon 21 of the PDE6B gene. Cytogenet Cell Genet. 2000;90:261-267.

21. Kukekova AV, Goldstein O, Johnson JL, et al. Canine RD3 mutation establishes rod-cone dysplasia type $2(r c d 2)$ as orthologue of human and murine rd3. Mamm Genome. 2009;20:109-123.

22. Petersen-Jones SM, Entz DD, Sargan DR. cGMP phosphodiesterase- $\alpha$ mutation causes progressive retinal atrophy in the Cardigan Welsh Corgi dog. Invest Ophthalmol Vis Sci. 1999;40(8):1637-1644.

23. Petersen-Jones SM, Zhu FX. Development and use of a polymerase chain reaction-based diagnostic test for the causal mutation of progressive retinal atrophy in Cardigan Welsh Corgis. Am J Vet Res. 2000;61: 844-846.

24. Dekomien G, Epplen JT. Exclusion of the PDE6A gene for generalised progressive retinal atrophy in 11 breeds of dog. Anim Genet. 2000;31:135-139.

25. Wang W, Acland GM, Ray K, Aguirre GD. Evaluation of cGMPphosphodiesterase (PDE) subunits for causal association with rod-cone dysplasia 2 (rcd2), a canine model of abnormal retinal cGMP metabolism. Exp Eye Res. 1999;69:445-453.

26. Petersen-Jones SM, Entz DD. An improved DNA-based test for detection of the codon 616 mutation in the alpha cyclic GMP phosphodiesterase gene that causes progressive retinal atrophy in the Cardigan Welsh Corgi. Vet Ophthalmol. 2002;5(2):103-103.

27. Downs LM, Bell JS, Freeman J, Hartley C, Hayward LJ, Mellersh CS Late-onset progressive retinal atrophy in the Gordon and Irish Setter breeds is associated with frameshift mutation in C2orf71. Anim Genet. 2012;44(2):169-177.

28. Lippmann T, Jonkisz A, Dobosz T, Petrasch-Parwez E, Epplen JT, Dekomien G. Haplotype-defined linkage region for gPRA in Schapendoes dogs. Mol Vis. 2007;13:174-180.

29. Dekomien G, Vollrath C, Petrasch-Parwez E, et al. Progressive retinal atrophy in Schapendoes dogs: mutation of the newly identified CCDC66 gene. Neurogenetics. 2010;11(2):163-174.

30. Gerding WM, Schreiber S, Schulte-Middelmann T, et al. Ccdc66 null mutation causes retinal degeneration and dysfunction. Hum Mol Genet. 2011;20(18):3620-3631.

31. Parshall CJ, Wyman M, Nitroy S, Acland GM, Aguirre GD. Photoreceptor dysplasia: an inherited progressive retinal atrophy of miniature schnauzer dogs. Prog Vet Comp Ophthalmol. 1991;1:187-203.

32. Zhang Q, Acland GM, Parshall CJ, et al. Characterization of canine photoreceptor phosducin cDNA and identification of a sequence variant in dogs with photoreceptor dysplasia. Gene. 1998;215(2):231-239.

33. Acland GD, Aguirre GM. Retinal degeneration in the dog IV. Early retinal degeneration (erd) in Norwegian elkhounds. Exp Eye Res. 1987; 44:491-521.

34. Goldstein O, Kukekova AV, Aguirre GD, Acland GM. Exonic SINE insertion in STK38L causes canine early retinal degeneration (erd). Genomics. 2010;96:362-368.

35. Downs LM, Wallin-Håkansson B, Boursnell M, et al. A frameshift mutation in golden retriever dogs with progressive retinal atrophy endorses SLC4A3 as a candidate gene for human retinal degeneration. PLoS One. 2011;6(6):e21452.

36. Downs LM, Mellersh CS. An intronic SINE insertion in FAM161A that causes exon-skipping is associated with progressive retinal atrophy in Tibetan Spaniels and Tibetan Terriers. PLoS One. 2014;9(4):e93990.

37. Ahonen SJ, Arumilli M, Lohi H. A CNGB1 frameshift mutation in Paillon and Phalène dogs with progressive retinal atrophy. PLoS One. 2013;8(8):e72122.

38. Winkler PA, Ekenstedt KJ, Occelli LM, et al. A large animal model for CNGB1 autosomal recessive retinitis pigmentosa. PLoS One. 2013;8(8):e72229.

39. Goldstein O, Jordan JA, Aguirre GD, Acland GM. A non-stop S-antigen gene mutation is associated with late onset hereditary retinal degenerations in dogs. Mol Vis. 2013;19:1871-1884.

40. Aguirre GD, Acland GM. Variation in retinal degeneration phenotype inherited at the prcd locus. Exp Eye Res. 1988;46(5):663-687. 
41. Acland GM, Halloran-Blanton S, Boughman JA, Aguirre GD. Segregation distortion in inheritance of progressive rod-cone degeneration (pred) in miniature-poodle dogs. Am J Med Genet. 1990;35(3): 354-359.

42. Aguirre GD, Alligood J, O'Brien P, Buyukmihci N. Pathogenesis of progressive rod-cone degeneration in miniature poodles. Invest $O p h-$ thalmol Vis Sci. 1982;23:610-630.

43. Acland GM, Ray K, Mellersh CS, et al. Linkage analysis and comparative mapping of canine progressive rod-cone degeneration (prcd) establishes potential locus homology with retinitis pigmentosa (RP17) in humans. Proc Natl Acad Sci USA. 1998;95(6):3048-3053.

44. Zangerl B, Goldstein O, Philp AR, et al. Identical mutation in a novel retinal gene causes progressive rod-cone degeneration in dogs and retinitis pigmentosa in humans. Genomics. 2006;88(5):551-563.

45. Dostal J, Hrdlicova A, Horak P. Progressive rod-cone degeneration (PRCD) in selected dog breeds and variability in its phenotype expression. Vet Med. 2011;56(5):243-247.

46. Kijas JW, Miller BJ, Pearce-Kelling SE, Aguirre GD, Acland GM. Canine models of ocular disease: outcross breedings define a dominant disorder present in the English mastiff and bull mastiff dog breeds. $J$ Hered. 2003;94(1):27-30.

47. Acland GM, Blanton S, Hershfield B, Aguirre GD. XLPRA: a canine retinal degeneration inherited as an X-linked trait. Am J Med Genet. 1994;52:27-33.

48. Zeiss CJ, Ray K, Acland GM, Aguirre GD. Mapping of X-linked progressive retinal atrophy (XLPRA), the canine homolog of retinitis pigmentosa 3 (RP3). Hum Mol Genet. 2000;9(4):531-537.

49. Zeiss CJ, Acland GM, Aguirre GD, Ray K. TIMP-1 expression is increased in $\mathrm{X}$-linked progressive atrophy despite its exclusion as a candidate gene. Gene. 1998;225:67-75.

50. Zhang Q, Acland GM, Wu WX, et al. Different $R P G R$ exon ORF15 mutations in Canids provide insights into photoreceptor cell degeneration. Hum Mol Genet. 2002;11(9):993-1003.

51. Beltran WA, Wen R, Acland GM, Aguirre GD. Intravitreal injection of ciliary neurotrophic factor (CNTF) causes peripheral remodeling and does not prevent photoreceptor loss in canine RPGR mutant retina. Exp Eye Res. 2007;84:753-771.

52. Beltran WA, Cideciyan AV, Lewin AS, et al. Gene therapy rescues photoreceptor blindness in dogs and paves the way for treating human X-linked retinitis pigmentosa. Proc Natl Acad Sci U S A. 2012;109(6): 2132-2137.

53. Vilboux T, Chaudieu G, Jeannin P, et al. Progressive retinal atrophy in the Border Collie: a new XLPRA. BMC Vet Res. 2008;4:10

54. Turney C, Chong NHV, Alexander RA, et al. Pathological and electrophysiological features of a canine cone-rod dystrophy in the miniature longhaired dachshund. Invest Ophthalmol Vis Sci. 2007;48(9): 4240-4249.

55. Mellersh CS, Boursnell MEG, Pettit L, et al. Canine RPGRIP1 mutation establishes cone-rod dystrophy in miniature longhaired dachshunds as a homologue of human Leber congenital amaurosis. Genomics. 2006;88:293-301.

56. Kuznetsova T, Iwabe S, Boesze-Battaglia $\mathrm{K}$, et al. Exclusion of RPGRIPI from primary causal association with early onset cone-rod dystrophy in dogs. Invest Ophthalmol Vis Sci. 2012;53(9):5486-5501.

57. Miyadera K, Kato K, Boursnell M, Mellersh CS, Sargan DR. Genomewide association study in RPGRIP1 (-/-) dogs identifies a modifier locus that determines the onset of retinal degeneration. Mamm Genome. 2012;23(1-2):212-223.

58. Ropstad EO, Bjerkås E, Narfström K. Clinical findings in early onset cone-rod dystrophy in the standard wire-haired dachshund. Vet Ophthalmol. 2007;10(2):69-75.

59. Wiik AC, Wade C, Biagi T, et al. A deletion in nephronophthisis 4 (NPHP4) is associated with recessive cone-rod dystrophy in standard wire-haired dachshund. Genome Res. 2008;18:1415-1421.

60. Kijas JW, Zangerl B, Miller B, et al. Cloning of the canine ABCA4 gene and evaluation in canine cone-rod dystrophies and progressive retinal atrophies. Mol Vis. 2004;10:223-232.
61. Goldstein O, Mezey JG, Schweitzer PA, et al. IQCB1 and PDE6B mutations causes similar early onset retinal degenerations in two closely related terrier dog breeds. Invest Ophthalmol Vis Sci. 2013;54: 7005-7019.

62. Goldstein O, Mezey JG, Boyko AR, et al. An ADAM9 mutation in canine cone-rod dystrophy 3 establishes homology with human conerod dystrophy 9. Mol Vis. 2010;16:1549-1569.

63. Kropatsch R, Petrasch-Parwez E, Seelow D, et al. Generalized progressive retinal atrophy in the Glen of Imaal Terrier is associated with a deletion in the ADAM9 gene. Mol Cell Probes. 2010;24(6):357-363.

64. Michaelides M, Hunt DM, Moore AT. The cone dysfunction syndromes. Br J Ophthalmol. 2004;88:291-297.

65. Sidjanin DJ, Lowe JK, McElwee JL, et al. Canine CNGB3 mutations establish cone degeneration as orthologous to the human achromatopsia locus ACHM3. Hum Mol Genet. 2002;11(16):1823-1833.

66. Yeh CY, Goldstein O, Kukekova AV, et al. Genomic deletion of CNGB3 is identical by descent in multiple canine breeds and causes achromatopsia. BMC Genet. 2013;14:27.

67. Komáromy AM, Alexander JJ, Rowlan JS, et al. Gene therapy rescues cone function in congenital achromatopsia. Hum Mol Genet. 2010; 19(13):2581-2593.

68. Lorenz B, Preising MN. Best's disease: overview of pathology and its causes. Ophthalmolge. 2005;102:111-115.

69. Guziewicz KE, Zangerl B, Lindauer SJ, et al. Bestrophin gene mutations causes canine multifocal retinopathy: a novel animal model for best disease. Invest Ophthalmol Vis Sci. 2007;48(5):1959-1967.

70. Zangerl B, Wickström K, Slavik J, et al. Assessment of canine BEST1 variations identifies new mutations and establishes an independent bestrophinopathy model (cmr3). Mol Vis. 2010;16:2791-2804.

71. Hoffman I, Guziewicz KE, Zangerl B, Aguirre GD, Mardin CY. Canine multifocal retinopathy in the Australian Shepherd: a case report. Vet Ophthalmol. 2012;15:134-138.

72. Gornik KR, Pirie CG, Duker JS, Boudrieau RJ. Canine multifocal retinopathy caused by a BEST1 mutation in a Boerboel. Vet Ophthalmol. 2014;17(5):368-372.

73. Guziewicz KE, Slavik J, Lindauer SJP, Aguirre GD, Zangerl B. Molecular consequences of BEST1 gene mutations in canine multifocal retinopathy predict functional implications for human bestrophinopathies. Invest Ophthalmol Vis Sci. 2011;52(7):4497-4505.

74. Pugh CR, Miller WW. Retinal and skeletal dysplasia in the Labrador retriever. Vet Med. 1995;90(6):593-596.

75. Goldstein O, Guyon R, Kukekova A, et al. COL9A2 and COL9A3 mutations in canine autosomal recessive oculo-skeletal dysplasia. Mamm Genome. 2010;21(7-8):398-408.

76. Palanova A. Collie eye anomaly: a review. Vet Med. 2015;60(7):345-350.

77. Cooper AE, Ahonen S, Rowlan JS, et al. A novel form of progressive retinal atrophy in Swedish vallhund dogs. PLoS One. 2014;9(9): e106610.

78. Akhmedov NB, Piriev NI, Ray K, Acland GM, Aguirre GD, Farber DB. Structure and analysis of the transducin beta3-subunit gene, a candidate for inherited cone degeneration (cd) in the dog. Gene. 1997;194(1):47-56.

79. Ray K, Acland GM, Aguirre GD. Nonallelism of erd and pred and exclusion of the canine RDS/peripherin gene as a candidate for both retinal degeneration loci. Invest Ophthalmol Vis Sci. 1996;37(5):783-794.

80. Davidson MG, Geoly FJ, Gilger BC, McLellan GJ, Whitley W. Retinal degeneration associated with vitamin E deficiency in hunting dogs. J Am Vet Med Assoc. 1998;213(5):645-651.

81. Itoh Y, Maehara S, Yamasaki A, Tsuzuki K, Izumisawa Y. Investigation of fellow eye of unilateral retinal detachment in Shi-Tzu. Vet Ophthalmol. 2010;13(5):289-293.

82. Grahn BH, Storey ES, McMillan C. Inherited retinal dysplasia and persistent hyperplastic primary vitreous in Miniature Schnauzer dogs. Vet Ophthalmol. 2004;7(3):151-158.

83. Robert L, Sénéchal A, Bocquet B, et al. Screening for canine model of choroideremia exclusively identifies nonpathogenic $\mathrm{CHM}$ variants. Ophthalmic Res. 2011;45:155-163. 
84. Schuh JCL. Secondary retinoschisis in a dog. J Comp Pathol. 1995;113(1):81-84.

85. Akhmedov NB, Piriev NI, Pearce-Kelling S, Acland GM, Aguirre GD, Farber DB. Canine cone transducin-gamma gene and cone degeneration in the $c d$ dog. Invest Ophthalmol Vis Sci. 1998;39:1775-1781.

86. Sancho-Pelluz J, Arango-Gonzalez B, Kustermann S, et al. Photoreceptor cell death mechanisms in inherited retinal degeneration. Mol Neurobiol. 2008;38(3):253-269.

87. Cvekl A, Mitton KP. Epigenetic regulatory mechanisms in vertebrate eye development and disease. Heredity. 2010;105(1):135-151.

88. Genini S, Guziewicz KE, Beltran WA, Aguirre GD. Altered miRNA expression in canine retinas during normal development and in models of retinal degeneration. Genomics. 2014;15:172.

89. Ray K, Baldwin VJ, Acland GM, Aguirre GD. Molecular diagnostic tests for ascertainment of genotype at the rod cone dysplasia $1(\mathrm{rcd} 1)$ locus in Irish Setters. Curr Eye Res. 1995;14(3):243-247.
90. Bechynova R, Dostal J, Stratil A, Jílek F, Horák P. Mutation in the RPE65 gene causing hereditary retinal dystrophy in the Briard dogs: application of a new detection method. Czech J Anim Sci. 2008;53(4):176-179.

91. Ray K, Tejero MD, Baldwin VJ, Aguirre GD. An improved diagnostic test for rod cone dysplasia 1 (rcd1) using allele-specific polymerase chain reaction. Curr Eye Res. 1996;15(5):583-587.

92. Mellersh C. DNA testing and domestic dogs. Mamm Genome. 2012; 23:109-123.

93. Miyadera K, Acland GM, Aguirre GD. Genetic and phenotypic variations of inherited retinal diseases in dogs: the power of within- and across-breed studies. Mamm Genome. 2012;23(0):40-61.

94. Leroy G. Genetic diversity, inbreeding and breeding practices in dogs: results from pedigree analyses. Vet J. 2011;189:177-182.

\section{Publish your work in this journal}

Veterinary Medicine: Research and Reports is an international, peer-reviewed, open access journal publishing original research, case reports, editorials, reviews and commentaries on all areas of veterinary medicine. The manuscript management system is completely online and includes a very quick and fair peer-review system.
Visit http://www.dovepress.com/testimonials.php to read real quotes from published authors. 